\title{
Identification of Heap-Carried Data Dependence Via Explicit Store Heap Models ${ }^{\star}$
}

\author{
Mark Marron $^{1}$, Darko Stefanovic ${ }^{1}$, Deepak Kapur ${ }^{1}$, and Manuel Hermenegildo ${ }^{1,2}$ \\ ${ }^{1}$ University of New Mexico \\ \{marron, darko, kapur\} @cs. unm. edu \\ 2 Technical University of Madrid and IMDEA-Software \\ herme@fi.upm.es
}

\begin{abstract}
Dependence information between program values is extensively used in many program optimization techniques. The ability to identify statements, calls and loop iterations that do not depend on each other enables many transformations which increase the instruction and thread-level parallelism in a program. When program variables contain complex data structures including arrays, records, and recursive data structures, the ability to precisely model data dependence based on heap structure remains a challenging problem.

This paper presents a technique for precisely tracking heap based data dependence in non-trivial Java programs via static analysis. Using an abstract interpretation framework, the approach extends a shape analysis technique based on an existing graph model of heaps, by integrating read/write history information and intelligent memoization. The method has been implemented and its effectiveness and utility are demonstrated by computing detailed dependence information for two benchmarks (Em3d and BH from the JOlden suite) and using this information to parallelize the benchmarks.
\end{abstract}

\section{Introduction}

The concept of data dependence between program statements is a fundamental tool for the reordering of program statements and the determination of invariant values in basic blocks, loops, or methods. Knowledge of data dependence allows the introduction of instruction-level parallelism and thread-level parallelism (both in loops and method invocations). In past work effective techniques for computing data dependence between scalar variables have been developed. However, the extension of this work to tracking memory-carried data dependence has been much less successful, in large part due to the lack of suitable heap analysis techniques to support them.

Previous work focused broadly on two approaches for identifying possible heapcarried data dependence, shape or points-to analysis as a proxy for data dependence [2, 4, 7, 14] wherein the identification of various acyclic structures and/or access path information is used to infer which expressions cannot access the same portion of the heap, and the explicit tracking of read/written locations [3, 8, 9] which model the set of locations that may be read/written at each program point. This work introduced several

* This work is supported in part by NSF grant 0540600. 
fundamental concepts involved in modeling heap carried data dependence. However experimental work with these approaches was limited to small numbers of microbenchmarks or used coarse points-to style analysis.

This paper builds on the basic concepts developed in earlier work and makes several contributions which are critical to analyzing non-trivial programs. The first is a novel method for tracking read/write locations during the analysis. The approach presented in this paper only tracks a two program locations per object field (one read location and one write location) instead of a set of all possible read locations and a set of all possible write locations per field. This is sufficient to identify the most recent program point where each memory location may be used/modified while avoiding the additional space usage and computational cost of tracking a set of program locations per object field. The next contribution is a method to efficiently track read/write information through method boundaries, in particular how to ensure that the addition of use-mod information does not have a serious impact on the memoization of method body analysis results, which is critical to applying the technique to realistic programs.

Our analysis technique uses an explicit store model for the heap objects which allows us to easily track the identity of objects between program statements. This differs from some recent work on shape analysis, which uses logical models with implicit store representations [5, 15] that cannot be efficiently extended to track the properties of arbitrary heap locations. It also differs from approaches based on separation logic which restrict the program to regular recursive structures and limited sharing of objects on the heap in order to ensure termination $[1,6]$. These features preclude the use of these approaches on many realistic application programs including the em3d and bh benchmarks, which we analyze as detailed case studies here.

\section{Running Examples}

We use examples in this paper to illustrate the various aspects of the analysis technique. The first is a small fragment created solely to illustrate the basics of the analysis. The second is a routine taken from em3d, one of the JOlden [10, 13] benchmarks.

The first example 1 creates 2 Data objects, each of which has a single integer field val, and puts them in a Pair object. If the conditional holds the first element of the pair is modified and then the swap method is called to interchange the first and second elements of the pair. This example is simple but relevant since in order to determine that the asserted property always holds the analysis needs to be able to track how pointer stores affect reachability relations in the heap, to identify where each heap location may be written, and do so across method invocations.

The second program fragment is a method taken from the em3d benchmark. This program builds a bipartite heap structure. Each call to computeNewValue takes a ENode object from one side of the bipartite graph and updates the value field of this node based on the value fields of ENode objects on the opposite side of the bipartite graph. This example demonstrates the importance of precisely resolving the heap structure so the dependence analysis can determine that the set of heap location where the value field is written is distinct from the locations that are read. 


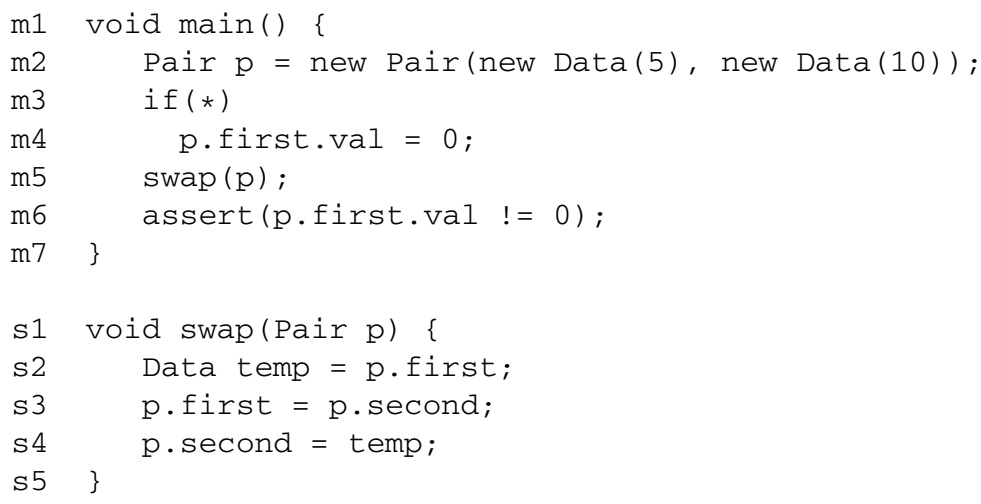

Fig. 1. Conditional Modify and Swap

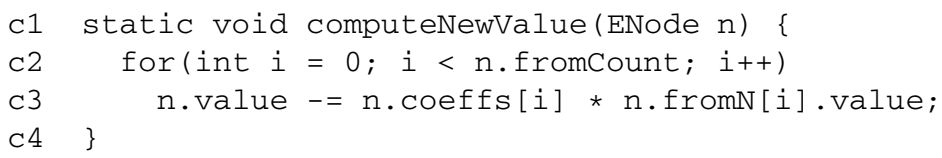

Fig. 2. Compute (From em3d)

\section{Abstract Heap Domain}

The underlying abstract heap domain that we extend is a graph in which each node represents a region of the heap (a set of objects or data structures) or a variable and each edge represents a set of pointers or a variable target. The nodes and edges are augmented with additional instrumentation predicates.

Types. Since each node in the graph represents a region of the heap (which may contain objects of many types) we use a set of type names for each node in the heap graph which contains the type of any object that may be in the region of the heap that is abstracted by the given node.

Linearity. To model the number of objects abstracted by a given node (or pointers by an edge) we use a linearity property which has 2 possible values 1 , which indicates that the node (edge) concretizes to either 0 or 1 objects (pointers) and the value $\omega$, which indicates that the node (edge) concretizes to any number of objects (pointers) in the range $[0, \infty)$.

Abstract Layout. To track the connectivity and shape of the region a node abstracts, the analysis uses abstract layout predicates Singleton, List, Tree, MultiPath, or Cycle. The Singleton predicate states that there are no pointers between any of the objects represented by an abstract node. The List predicate states that each object has at most one pointer to another object in the region. The other predicates correspond to the standard definitions for Trees, Dags, and Cycles in the literature. 
Interference. The heap model uses two properties to track the potential that multiple pointers or variables can reach the same memory location in the region that a particular node represents. In this work the examples only require one of these properties (interference) so we omit the discussion of the other property (connectivity) and refer the interested reader to [12] for a more detailed description.

Each edge abstracts a set of pointers in the concrete program. The interfere property has three possible values, to track that some of the pointers may alias ( $a p)$, that none of the pointers alias but they may point into the same data structure (thus can interfere, $i p$ ), or that each of the pointers refers to a unique and disjoint data structure in the node that the edge ends at (they are disjoint and non-interfering, $n p$ ).

Heap Representation. We represent abstract heaps pictorially as labeled, directed multigraphs. The variable nodes are labeled with the variable that they represent. The nodes representing the regions are represented as a record [type, linearity, layout] that tracks the instrumentation predicates.

The edges (which represent sets of pointers) in the figures are represented as records [offset, linearity, interfere]. The offset component indicates the offsets (labels) of the references that are abstracted by the edge. These labels may be any of the field identifiers that are used in the program or the special label, ?, which is the label given to the summary field representing all the elements in a collection object Vector, List, or an array.

To simplify the figures we omit entries in the labels when they are the default domain value. The default values for the nodes are layout $=(S)$ ingleton and linearity $=1$. The default edge values are linearity $=1$ and interfere $=n p$. The variable edges always represent single references and the label is always implicitly the variable name.

\subsection{Heap Structure Examples}

Pair Example. Figure 3 shows the heap model (without any read/write information) that is computed as the result of executing the pair constructor in the first example program. The variable $\mathrm{p}$ points to a single object of type Pair (the linearity is 1 and the shape in Singleton, as described above this default information is omitted from the figure). The node representing the Pair object has 2 outgoing edges representing the two pointers stored in the first and second fields. The analysis determines that these edges each represent a single pointer (and since any edge representing a single pointer cannot have any interference the interfere property is $n p$ ). Again the default

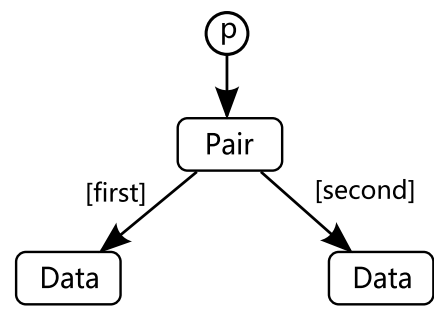

Fig. 3. Pair Allocation, Structure Only 
properties of linearity 1 and non-interference are omitted from the figure. Finally, the model shows that the first and second pointers each refer to a single Data object.

Em3d Example. The state of the heap at the entry to the computeNewValue method in the program em3d is shown in Figure 4(again without any read/write information). The em3d program computes electro-magnetic field values in a 3-dimensional space by constructing a list of ENode objects, each representing an electric field value and a second list of ENode objects, which represent a magnetic field values. To compute how the electric/magnetic field value for a given ENode object is updated at each step the computeNewValue method uses an array of ENode objects from the opposite field and performs a convolution of these field values and a scaling vector, updating the current field value with the result.

Figure 4 shows the heap structure computed for the computeNewValue method. We have placed dashed lines around the structures that represent the magnetic field (in blue if color is available) and the electric field (in green). Variable g points to a single object of type BiGrph, which is the data structure that encapsulates all the objects of interest. The BiGrph object has 2 fields, the hNodes field pointing to a linked list of ENode objects that make up the magnetic field and, the eNodes field pointing to a linked list of ENode objects that make up the electric field.

Looking at the structures in the magnetic field we see the edge labeled $[?, \omega]$ which represents all the pointers stored in the linked list. Since the linearity is $\omega$ we know the edge may represent multiple pointers but each of these pointers must point to a unique ENode object (the default interference value of non-interfering $n p$ is omitted). The figure also shows that the magnetic field is represented by many ENode objects (the node labeled [ENode, $\omega]$ ) each of which has a pointer to a unique array of floats

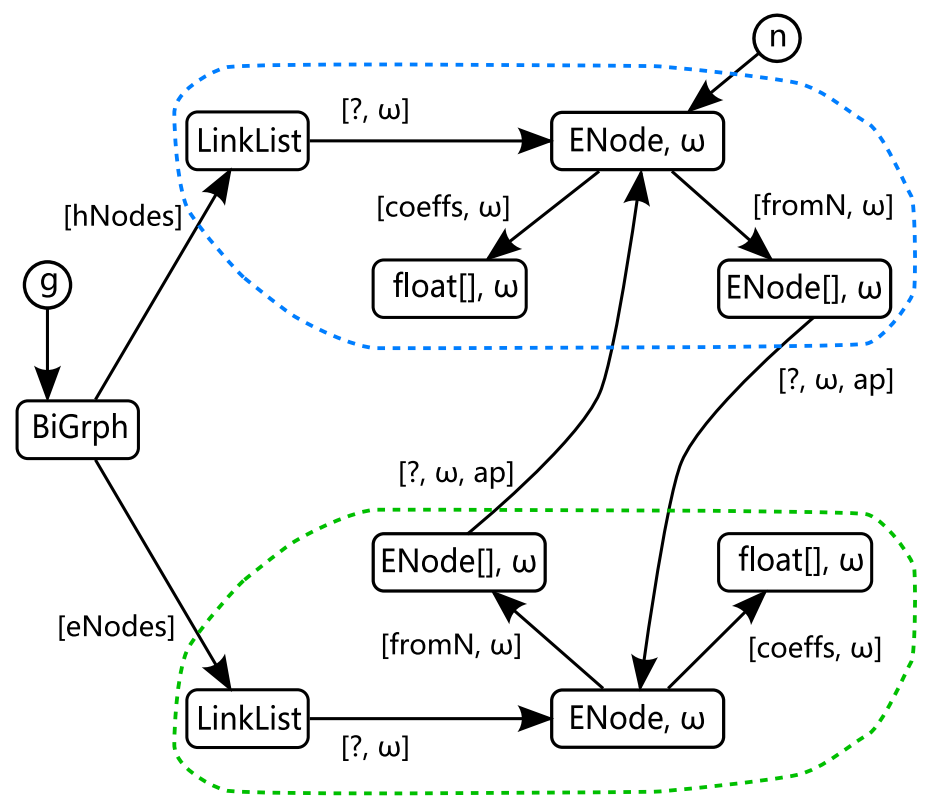

Fig. 4. computeNewValue, Structure Only 
(the edge labeled coeffs) and an array of ENode objects (the edge labeled fromN) which are used as the set of nodes from the opposite field. The edge that represents the pointers stored in this array is labeled [?, $\omega, a p]$, which indicates that it represents all the pointers stored in the array and, that the pointers it represents may alias (ap).

\section{Data Dependence Extensions}

To track the read/write histories of objects on the heap we extend the model presented in Section 3 with information to track the identity of the objects represented by a given node, and for each field in the object we track the most recent program location (statement or control flow structure) where a read/write of that field may have occurred.

In order to ensure that the initial shape analysis when augmented with the read/write domain remains efficient it is critical to minimize the amount of additional information that is added to the heap model. The key observation is that for most optimization applications the shape analysis only needs to provide precise information about the most recent program location at which each field may have been read or written. Thus, the analysis does not need to track every possible program location where a field may have been read/written, and this significantly reduces the computational requirements.

\subsection{Intermediate Representation}

Before we introduce the domain extensions we need to specify how program locations are represented. To simplify the analysis the Java programs are transformed into a structured mid-level intermediate language (called MIL). The partial grammar below provides a sample of the language constructs in the intermediate representation.

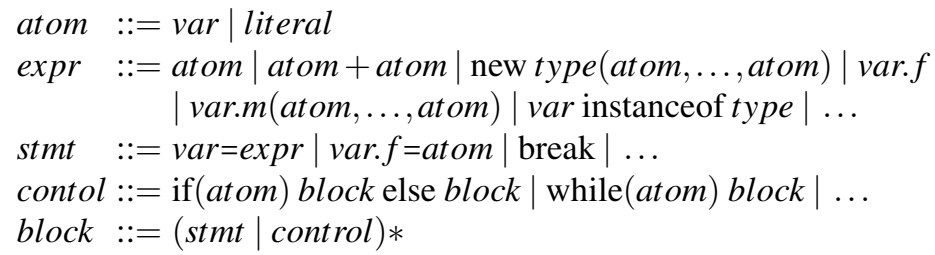

The language has method invocations, conditional constructs (if, switch), exception handling (try-throw-catch) and looping statements (for, do, while). The state modification and expressions cover the standard range of program operations (load, store and assign along with logical, arithmetic and comparison operators). We associate with each statement and each control flow structure a program location $\ell$.

\subsection{Extended Domain}

Read-Write Locations. Each node may represent a number of objects of different types $\left(\tau_{1} \ldots \tau_{m}\right)$ and each type may have many fields $\left(f_{\tau_{i}}^{1} \ldots f_{\tau_{i}}^{n}\right)$. For each of these fields we keep two program locations $(\ell)$, the last time the field may have been read $\left(\ell_{r}\right)$ and the last time the field may have been written $\left(\ell_{w}\right)$.

Node Identity. In order to efficiently analyze method invocations we memoize the input and return abstract states and reuse them as possible. In order to prevent spurious 


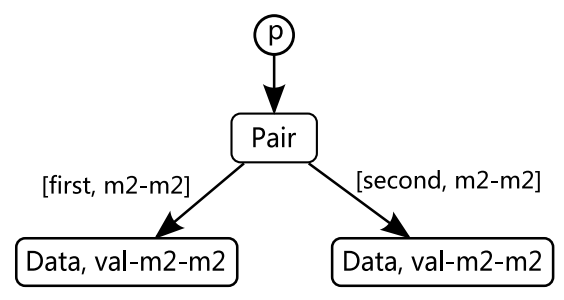

Fig. 5. Pair with use-mod

inequalities between the read/write program locations (that refer to the locations in the caller scope) in the memoized models we replace them with a generic modified outside value. To allow us to match the identities of the objects in the input state with their position in the output state we add a unique identity tag (a value in $\mathbb{N}$ ) to each node that is passed into a method call.

In our extended domain each node in the heap is now represented as a tuple [type, linearity, layout, scalar-fields, identity] . The entries type, layout and count are as described in Section 3. The scalar-fields entry is a list of field-readloc-writeloc entries, one for each scalar field, where readloc and writeloc are either a program location $\ell$ or the special entry 0 (modified outside). The identity entry is a set of identity tags or is omitted entirely if the node does not have a identity tag associated with it (or for clarity if it is not relevant to the example).

To track the read write information for the pointer fields we extend each edge label to [offset, linearity, interfere, readloc-writeloc] where readloc and writeloc are defined the same as for the scalar fields in the nodes. Again, for clarity, we omit readloc-writeloc information if it is irrelevant to the example.

Figure 5 shows the model that is computed as the result of executing the pair constructor in the first example program. The pair is marked as having read and written the two pointer fields at initialization (the $m 2-m 2$ entries on the first and second edges) and the identity tag is omitted (since this object was allocated in the current scope). The two Data objects which had their val fields initialized at program location $m 2$ have the entry $m 2-m 2$ in their scalar-fields read/write entry.

\subsection{Local Data Dependence}

Now that we have extended the model with the required instrumentation properties we can define a set of dataflow operations to model the effects of program operations on the read/write information. The changes for load and store operations are simple, only requiring an update of the last read/write value for the target object to the current program location, thus we omit a detailed description of these operations.

Data Flow Domain. If $\mathscr{G}$ is the set of all possible heap graphs and $\mathscr{B}=\wp($ var $) \times$ $\wp($ var) (a simple domain to track which variables must be true, the first element of the pair, and which must be false, the second element) then our abstract domain is $\mathscr{D}=\wp(\mathscr{G} \times \mathscr{B})$. 
Given an element in the abstract domain, $\sigma \in \mathscr{D}$, we assume the abstract semantics are defined for expressions and statements. Thus, given an expression $e$, the abstract semantics of this expression on the abstract state $\sigma$ are given by $\mathscr{S} \llbracket e \rrbracket \sigma$ and similarly for statement $s$, the abstract semantics are given by $\mathscr{S} \llbracket s \rrbracket \sigma$. Using the $\mathscr{B}$ component of the domain and a boolean condition $b$, we can filter an abstract state $\sigma=\left\{\theta_{1}, \ldots, \theta_{k}\right\}$ into two new abstract states $\sigma_{\text {true }}=\mathscr{S} \llbracket b \rrbracket_{\text {true }}(\sigma)=\left\{\theta_{i} \mid \mathrm{b}\right.$ may be true in $\left.\theta_{i}\right\}$ and $\sigma_{\text {false }}=$ $\mathscr{S} \llbracket b \rrbracket_{\text {false }}(\sigma)=\left\{\theta_{i} \mid \mathrm{b}\right.$ may be false in $\left.\theta_{i}\right\}$.

Abstract Conditional Semantics. Using the above definitions we can write the standard definition for the if statement, $\mathscr{S} \llbracket$ if $(b)$ block $_{t}$ else block $f \rrbracket \sigma=\mathscr{S} \llbracket$ block $_{t} \rrbracket\left(\sigma_{\text {true }}\right) \cup$ $\mathscr{S} \llbracket$ block $_{f} \rrbracket\left(\sigma_{\text {false }}\right)$. However, using this definition of the semantics can result in exponential growth in the number of states that the analysis must deal with (since for most cases at the union of the abstract states that result from analyzing true and false branches will have many models that are identical except for a few readloc-writeloc entries).

To avoid this we replace all the readloc-writeloc entries that refer to program locations in the true or false branches of the conditional with the program location of the conditional before the union operation. Thus any differences that are solely due to readloc-writeloc entries are removed and exponential growth is avoided. Given $\sigma=$ $\left\{\theta_{1}, \ldots, \theta_{k}\right\}$ and a block which contains statements/control structures at program locations $p l=\left\{v_{1}, \ldots, v_{i}\right\}$, we define the operator $\boldsymbol{s}(\sigma$, block, $\mu)=\left\{\left.\theta_{i}\right|_{p l} ^{\mu} \mid \theta_{i} \in \sigma\right\}$, which performs the required replacements in the heap graph models. With this definition the improved semantics for the conditional operation (at program location $\kappa$ ) are:

$$
\begin{aligned}
& \mathscr{S} \llbracket \text { if }(\text { b }) \text { block }_{t} \text { else block }_{f} \rrbracket \sigma= \\
& \quad \mathbf{Q}\left(\mathscr{S} \llbracket \text { block }_{t} \rrbracket\left(\sigma_{\text {true }}\right), \text { block }_{t}, \kappa\right) \cup \mathbf{S}\left(\mathscr{S} \llbracket \text { block }_{f} \rrbracket\left(\sigma_{\text {false }}\right), \text { block }_{f}, \kappa\right)
\end{aligned}
$$

Disjunctive Domain. To speed program analysis we employ a partially disjunctive domain [11] which we use to discard elements in the abstract states $\left(\theta_{i}\right)$ that contain redundant read/write information. This is done by defining an order on the program locations based on their control-flow order. In general this order is not total (e.g. statement locations in the true and false branches of an if statement). However, our replacement of locations inside nested control-flow structures with the program location of the structure that contains them ensures that we can always compare the program locations that appear in the readloc-writeloc entries.

Analyze Conditional Example. Figure 6(a) is the abstract heap that approximates the state of the program after the true branch $\left(\mathscr{S} \llbracket\right.$ block $\left._{t} \rrbracket\left(\sigma_{\text {true }}\right)\right)$, where the first element of the pair had the val field written. In the node that represents the Data object that was written we updated the writeloc entry to program location $m 4$ (where the write occurred, marked in red if color is available). Figure 6(b) shows the result of \$ $\left(\mathscr{S} \llbracket\right.$ block $_{t} \rrbracket\left(\sigma_{\text {true }}\right)$, block $\left._{t}, m 3\right)$, where we replaced the readloc-writeloc locations that appear in the true branch with program location of the if statement (program location $m 3$, shown in blue).

Figure 6(c) shows the abstract heap from the false branch where no write occurred $\left(\mathscr{S} \llbracket\right.$ block $\left._{f} \rrbracket\left(\sigma_{\text {false }}\right)\right)$. The most recent $\bmod$ location is unchanged (program location $m 2$, where the object was initialized) in $\boldsymbol{\phi}\left(\mathscr{S} \llbracket\right.$ block $_{f} \rrbracket\left(\sigma_{\text {false }}\right)$, block $\left._{f}, m 3\right)$ since program location $m 2$ is not nested in the conditional. 


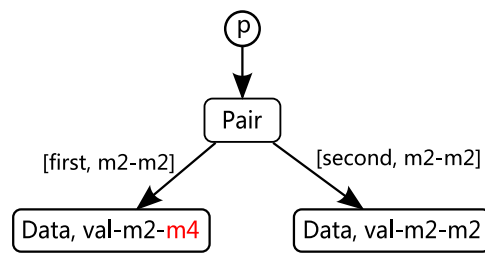

(a) True Branch

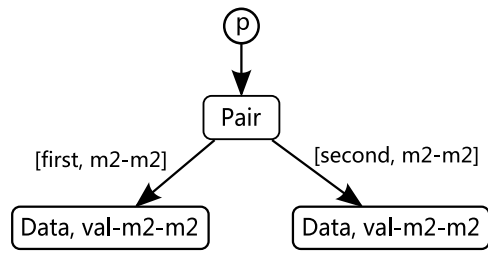

(c) False, After Mod Location Update

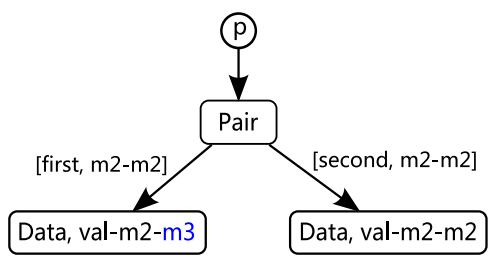

(b) True, After Mod Location Update

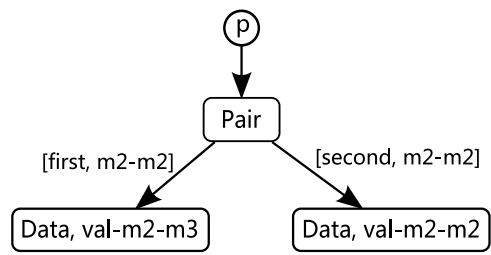

(d) Discard Subsumed False Branch

Fig. 6. Updating Read/Write Locations At Control Flow Join

Given our order relation on the use-mod sites we can simplify the models resulting from the true and false branches into a single model shown in Figure 6(d) Intuitively the may use-mod information from the true branch indicates that the memory location at p. first. val may have been written at location $m 3$ (the if statement) or at some previous point in the program, while the result of the false branch indicates that the memory location at p. first.val may have been written at location $m 2$. Since the possibility that the object may be written at or before program location $m 2$ is implied by the statement that the object may be written at or before program location $m 3$ we can safely discard the model from the false branch.

Abstract Loop Semantics. The semantics of a looping statement while at program location $\kappa$ can be expressed in terms of accumulating all possible exit states. To do this we define the state of the heap at the loop test for the $i^{\text {th }}$ iteration of the loop as:

$$
\sigma_{i}= \begin{cases}\sigma & \text { if } i=0 \\ \mathscr{S} \llbracket \text { block } \rrbracket\left(\mathscr{S} \llbracket b \rrbracket_{\text {true }}\left(\sigma_{i-1}\right)\right) & \text { otherwise }\end{cases}
$$

Then we can define the semantics of the loop analysis as the union of all the possible exits from the loop with the read/write program locations that occur within the loop body replaced by the program location of the loop $(\kappa)$. Formally:

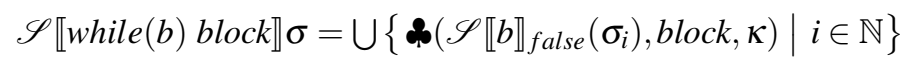

\subsection{Interprocedural Data Dependence}

In order to efficiently handle large programs we memoize results of analyzing each method. At method call sites, if we were to naively compare the memoized heap models with the current call state the method specific readloc-writeloc entries we embed in the model would create many spurious inequalities. As an example consider the swap 
function from our running example. The swap method could be called from multiple locations in a program and at each of these call sites the Pair object may have a different readloc-writeloc entries for the first and second fields. If comparision is done in a naive manner these differences will result in spurious mismatches with memoized analysis values, forcing the method to be re-analyzed for each call.

To avoid this problem we anonymize the readloc-writeloc locations before attempting to find a match in the memo table. However, when doing this anonymization we need to ensure that we can figure out which locations in the result heap may have been $\mathrm{read} / \mathrm{written}$ in the call and which must not have been read/written (and thus have the same readloc-writeloc entry as before the call).

Call Example. The anonymization and remapping operations are conceptually simple but without some intuition into how they function the definitions are difficult to follow. Thus, we first examine how the swap call is handled in the pair example. Figure 7 shows the steps that are taken to analyze the call at program location $m 5$ assuming that the memo table contains Subfigures 7(a) and 7(b) as a memoized result.

Figures 7(a) and 7(b) show that during the analysis of the swap method the analysis has determined the first and second fields have been read and written (the readloc and writeloc entries refer to program locations within the swap method, $s 2, s 3$ and $s 4$ ) but that the val fields are neither read nor written. The readloc and writeloc entries are the modified-outside value 0 . Further, based on the identity tag sets we know that the object which was stored in the first field at the method entry (Figure 7(a)) and was given the identity tag 2 is stored in the second field at the method exit (Figure 7(b)). A similar situation holds for the object stored in the second field at the method entry, which was assigned the identity tag 3 .

Figure $7(\mathrm{c})$ shows the state of the heap model at the call site (location $\mathrm{m5}$ ) after we have added fresh tags (7, 8, and 9) to uniquely identify the nodes. After anonymizing the locations of the readloc-writeloc entries to the modified-outside value (0) we have the model shown in Figure 7(d), which is isomorphic (up to identity tags) to the model in our memo table, Figure 7(a)

During the anonymization we construct a map from the identity tags we added and the field identifiers to the readloc-writeloc entries in the caller scope that we are anonymizing. This gives us the $\operatorname{map} \operatorname{Mod} M=\{(7$, first $) \rightarrow(m 2, m 2),(7$, second $) \rightarrow$ $(m 2, m 2),(8, \mathrm{val}) \rightarrow(m 2, m 3),(9, \mathrm{val}) \rightarrow(m 2, m 2)\}$. Using the isomorphism from $\sigma_{\text {in }} \mapsto \sigma_{\text {call }}$ we have a map $\Pi=\{1 \rightarrow 7,2 \rightarrow 8,3 \rightarrow 9\}$.

Using these maps we transfer the read/write information from the call input to the memoized output, replacing any readloc-writeloc entries that refer to program locations in the callee body (swap) with the program location of the call site (program location $\mathrm{m5}$ ) and replacing any occurrences of the modified outside value with the appropriate entry from $\bmod M$. In Figure 7(b) the node with identify tag 2 has the modified outside value for the readloc/writeloc of the val field (val-0-0). To place the correct readloc-writeloc values into this node we look up the node that it maps to in the caller scope (via the $\Pi$ map), which gives us the identity tag 8 . Then we look up the caller scope readloc-writeloc information in the $\bmod M$ map, which gives us the read/write information for the field, $\mathrm{m} 2-\mathrm{m} 3$. 


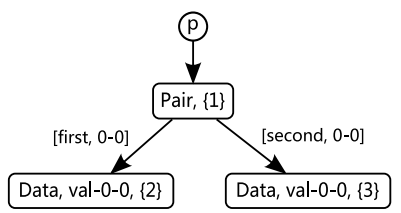

(a) Memo In

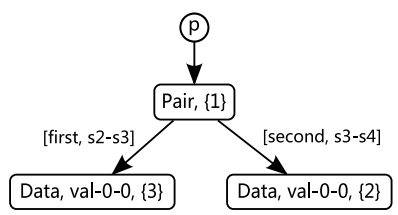

(b) Memo Out

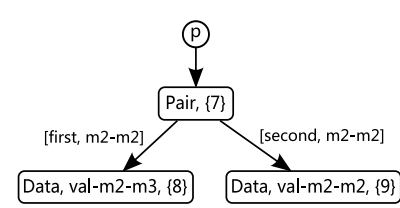

(c) Call In

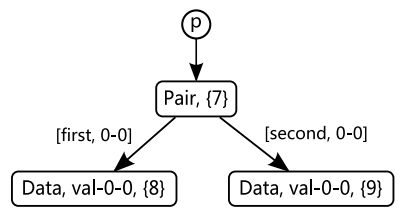

(d) Anonymized

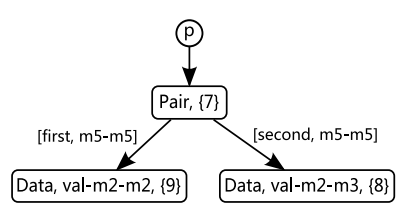

(e) Call Out

Fig. 7. Mapping Through Memoization

This remapping gives us the result in Figure 7(e), which shows that the object stored in the second field of the Pair object may have been written at program location $m 3$ but that the object stored in the first field has not been modified since initialization at program location $m 2$. Thus, we can determine that the read from p. first.val is non-zero and the assertion will always succeed.

Dataflow Operations. For a method invocation at call site $\ell_{\text {call }}$ we give each node in the call state $\sigma_{\text {call }}$ a unique tag $\kappa \in \mathbb{N}$, set the read/write location to the modified outside value and build a map ModM $: \mathbb{N} \times$ field $\mapsto\left(\ell_{r}, \ell_{w}\right)$.

We then compare the anonymized version of $\sigma_{\text {call }}$ with the entries in the memo table ignoring the read/write information. If a match $\left(\sigma_{\text {in }}, \sigma_{\text {out }}\right)$ is found then there is a graph isomorphism $\Phi: \sigma_{\text {in }} \mapsto \sigma_{\text {call }}$. This isomorphism and the fact that the set of location tags in $\sigma_{\text {in }}$ and $\sigma_{\text {out }}$ are the same implicitly defines a map, $\Pi:\left\{\kappa \mid \kappa\right.$ a location tag $\left.\in \sigma_{\text {out }}\right\}$ $\mapsto\left\{\kappa^{\prime} \mid \kappa^{\prime}\right.$ a location tag $\left.\in \sigma_{\text {call }}\right\}$. Using this map we can then compute the result of the call by replacing any readloc-writeloc values $\left(\ell_{x}\right)$ for the fields in each node $n$ with:

$$
\left(\ell_{x}^{\prime}\right)=\left\{\begin{array}{l}
\ell_{\text {call }}, \text { if } \ell_{x}^{\prime} \text { is a location in the callee method } \\
\max \left(\left\{n^{\prime} \cdot \ell_{x} \mid \kappa \in n . \text { identity } \wedge n^{\prime} \in \sigma_{\text {call }} \wedge \Pi(\kappa) \in n^{\prime} \text {.identity }\right\}\right), \text { otherwise }
\end{array}\right.
$$

\section{Experimental Results}

In this section we examine how the data dependence information can be used to perform thread level parallelization on variations of two of the more complex JOlden benchmarks, em3d and bh [10, 13]. To asses the performance of our approach we examine the analysis runtime on the JOlden suite, several of the SPECjvm98 benchmarks [16], and a logic formula manipulation program we developed as test case.

\subsection{Case Studies}

Em3d. The first application of the read/write dependence information we look at is performing thread-level parallelization of the em3d benchmark. In Figure 2 we show 


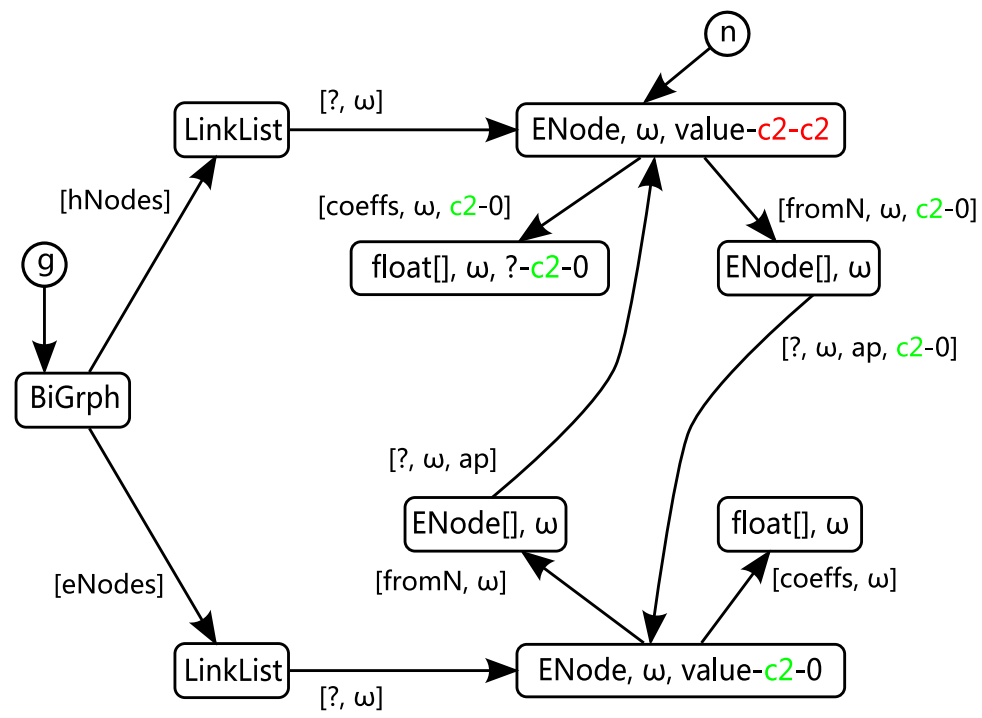

Fig. 8. Em3d With Read/Write Info

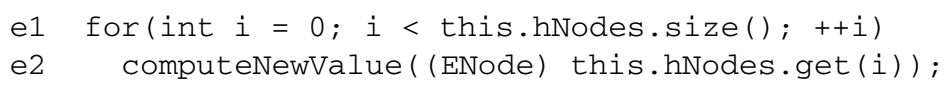

Fig. 9. Main Em3d Compute Loop

the code for updating the value field of a single ENode object. By applying our $\mathrm{read} / \mathrm{write}$ analysis we obtain the model in Figure 8 at the end of the method body. We see that some object from the list of magnetic field nodes has had the value field both read and written in the loop, readloc $=c 2$ and writeloc $=c 2$ (marked in red if color is available), while there have been reads from the coef $\mathrm{fs}$ and $\mathrm{fromN}$ pointer fields, readloc $=c 2$ (marked in green), writeloc $=0$. The pointers in the fromN array have also been read in order to access the value fields in the ENode objects in the opposite field, which have been read but not written ( readloc $=c 2$, writeloc $=0$ ).

Using this information, the fact that each reference in the linked list (LinkList) of ENode objects refers to a unique object (the edge is $n p$, the omitted default interference value) and the linear loop iteration, allows us to determine that each magnetic ENode object is written on a single iteration of the main update loop, program location $e 2$, in Figure 9 which calls computeNewValue. Given this information it is valid to thread parallelize this loop (and to vectorize the loop in computeNewValue). Doing so results in a speedup of 3.21 on our quad-core test machine.

$\mathrm{BH}$. Figure 10 shows the model that the analysis computes for the heap based read/write information in the hackGravity method of the Barnes-Hutt benchmark. For clarity we have simplified the heap structure in areas that are not relevant to this example.

The bh program performs a fast-multipole algorithm on the gravitational interaction between a set of bodies (the Body objects) and uses a space decomposition tree of 


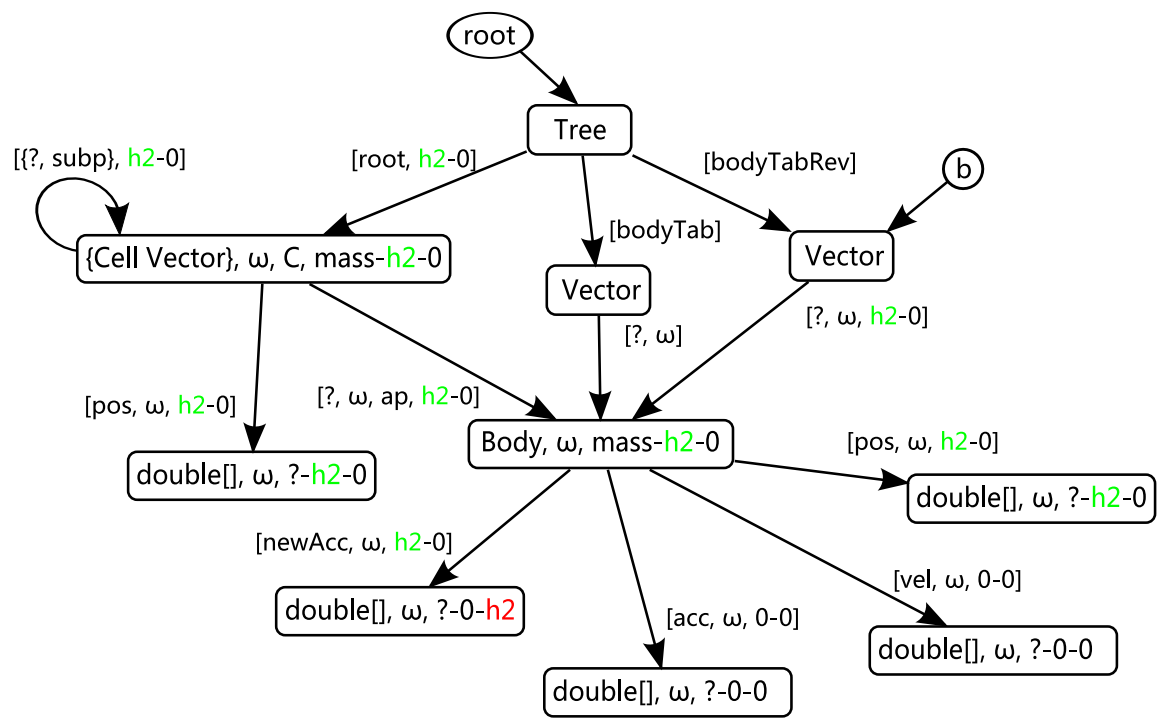

Fig. 10. BH With Read/Write Info

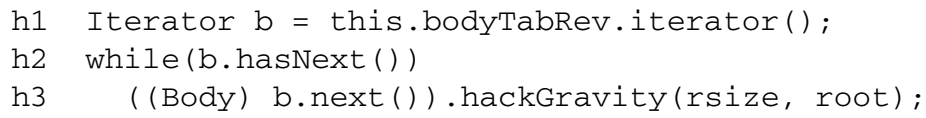

Fig. 11. Main Update, Gravity Computation

Cell objects each of which has a Vector containing a subtree or a reference to the Body objects. The program also keeps two vectors for accessing the bodies, bodyTab and bodyTabRev. Figure 10 shows the state of the heap model after the loop body (Figure 11) that contains the majority of the computation in bh. This loop takes each Body object and walks the space decomposition tree (the root field) to determine a new acceleration value for the Body object (stored in the newAcc field).

Our analysis is not able to precisely resolve the construction of the space decomposition tree and conservatively assumes it may be a cyclic structure (shown by the $\mathrm{C}$ in the node representing the Cell objects). However, the analysis is able to determine that the Cell objects and the Body objects represent distinct regions in the program. This piece of information combined with the observation that the space decomposition tree is only read in the loop body (all the readloc entries set to $h 2$, marked in green, and the writeloc entries set to 0 ), that the only part of the heap which is modified is never read (the double [ ] stored in the newAcc field, writeloc $=h 2$, set to red), and that the collection being indexed over (the Vector referred to by the bodyTabRev field) does not have multiple references to the same object (the ? edge is $n p$, the omitted default interference value), is sufficient to ensure that there are no heap-carried dependence in this loop. Thus, we can safely thread-parallelize the loop body, achieving a factor of 2.98 speedup on our test machine. 


\subsection{Performance}

The analysis algorithm was written in $\mathrm{C}++$ and compiled using gcc 4.2. The analysis as well as the parallelization benchmarks were run on a $2.6 \mathrm{GHz}$ Intel quad-core machine with 4 GB of RAM (although memory consumption never exceeded $60 \mathrm{MB}$ ).

The original Java programs are transformed into MIL programs and the required stub code is added to enable the analysis of the standard Java libraries (which requires from 200-600 lines depending on which libraries the benchmark uses). These MIL programs are then processed by the analyzer. A demo version of the analyzer and benchmarks can be obtained at [13].

\begin{tabular}{|l|l|l|l|l||r|r|}
\hline Benchmark & LOC & Classes & Methods & Analysis Time & Shape & RW Dep \\
\hline bisort & 560 & 36 & 348 & $0.26 \mathrm{~s}$ & $\mathrm{Y}$ & $\mathrm{Y}$ \\
mst & 668 & 52 & 485 & $0.12 \mathrm{~s}$ & $\mathrm{Y}$ & $\mathrm{Y}$ \\
tsp & 910 & 42 & 429 & $0.15 \mathrm{~s}$ & $\mathrm{Y}$ & $\mathrm{Y}$ \\
em3d & 1103 & 56 & 488 & $0.31 \mathrm{~s}$ & $\mathrm{Y}$ & $\mathrm{Y}$ \\
perimeter & 1114 & 44 & 381 & $0.91 \mathrm{~s}$ & $\mathrm{P}$ & $\mathrm{N}$ \\
health & 1269 & 59 & 534 & $1.25 \mathrm{~s}$ & $\mathrm{Y}$ & $\mathrm{Y}$ \\
voronoi & 1324 & 58 & 549 & $1.80 \mathrm{~s}$ & $\mathrm{Y}$ & $\mathrm{Y}$ \\
power & 1752 & 57 & 520 & $0.36 \mathrm{~s}$ & $\mathrm{Y}$ & $\mathrm{Y}$ \\
bh & 2304 & 61 & 576 & $1.84 \mathrm{~s}$ & $\mathrm{P}$ & $\mathrm{Y}$ \\
\hline db & 1985 & 68 & 562 & $1.42 \mathrm{~s}$ & $\mathrm{Y}$ & $\mathrm{Y}$ \\
logic & 3960 & 72 & 620 & $48.26 \mathrm{~s}$ & $\mathrm{P}$ & $\mathrm{Y}$ \\
raytrace & 5809 & 63 & 506 & $37.09 \mathrm{~s}$ & $\mathrm{Y}$ & $\mathrm{Y}$ \\
\hline
\end{tabular}

Fig. 12. LOC is the size of the program after transformation to MIL (including library stub code that must be analyzed), Classes/Methods are the number of classes/methods in the program (including Java Libraries that are used). Shape reports the heap connectivity is correctly identified and RW Dep reports if the RW information is useful (as in Section 5.1).

We report Y(es) in the Shape column if the analysis correctly identified all the relevant the shape information of the heap structures in the program. $\mathrm{P}($ artial $)$ means the analysis was able to determine the precise shape for some of the data structures but that some properties were missed.

We report similar information for the utility of the RW information. Y(es) means the $\mathrm{read} / \mathrm{write}$ information would be sufficient to introduce substantial thread level parallelism (as in Section 5.1) and provides the information required to enable significant instruction level parallelism optimizations (e.g. code motion to improve scheduling, elimination of redundant loads/stores or the identification of loop invariant values). We Report (N)o for only one of the benchmarks, perimeter, where the read/write information does not enable any thread level parallelism and only enables minor scheduling or load elimination opportunities.

Our experimental results show that the analysis is capable of efficiently computing very precise heap-carried dependence information over a range of benchmarks. In particular the ability to compute this information on the benchmarks bh, em3d, voronoi and raytrace is a significant advance in the state of the art for understanding the program heap. Computing precise shape and dependence information for these benchmarks 
requires the analysis to precisely model recursive data structures, Java collections, nontrivial sharing between components of the heap and, in order to compute the dependence information, to precisely track the part of the heap each read/write affects.

The analysis presented in this paper is not only capable of accurately modeling all of these features but is able to do so efficiently (analyzing the smaller benchmarks takes less than 2 s per benchmark and raytrace at 5809 LOC takes only 37s). Based on these results we believe that the analysis reported in this paper is robust enough to be generally useful in the optimization of smaller Java programs and we plan to continue work on scaling the analysis to handle larger programs with the same level of precision.

\section{References}

1. Berdine, J., Calcagno, C., Cook, B., Distefano, D., O’Hearn, P., Wies, T., Yang, H.: Shape analysis for composite data structures. In: Damm, W., Hermanns, H. (eds.) CAV 2007. LNCS, vol. 4590, pp. 178-192. Springer, Heidelberg (2007)

2. Cheng, B.-C., Mei, W., Hwu, W.: Modular interprocedural pointer analysis using access paths: design, implementation, and evaluation. ACM SIGPLAN Notices (2000)

3. Choi, J.-D., Burke, M., Carini, P.: Efficient flow-sensitive interprocedural computation of pointer-induced aliases and side effects. In: POPL (1993)

4. Ghiya, R., Hendren, L.J., Zhu, Y.: Detecting parallelism in C programs with recursive data structures. In: CC (1998)

5. Gulwani, S., Tiwari, A.: An abstract domain for analyzing heap-manipulating low-level software. In: CAV (2007)

6. Guo, B., Vachharajani, N., August, D.: Shape analysis with inductive recursion synthesis. In: PLDI (2007)

7. Hendren, L.J., Nicolau, A.: Parallelizing programs with recursive data structures. IEEE TPDS 1(1) (1990)

8. Horwitz, S., Pfeiffer, P., Reps, T.W.: Dependence analysis for pointer variables. In: PLDI (1989)

9. Hummel, J., Hendren, L.J., Nicolau, A.: A general data dependence test for dynamic, pointerbased data structures. In: PLDI (1994)

10. Suite, J.: http://www-ali.cs . umass . edu/DaCapo/benchmarks . html

11. Manevich, R., Sagiv, S., Ramalingam, G., Field, J.: Partially disjunctive heap abstraction. In: Giacobazzi, R. (ed.) SAS 2004. LNCS, vol. 3148, pp. 265-279. Springer, Heidelberg (2004)

12. Marron, M., Kapur, D., Stefanovic, D., Hermenegildo, M.: A static heap analysis for shape and connectivity. In: Almási, G.S., Caşcaval, C., Wu, P. (eds.) KSEM 2006. LNCS, vol. 4382, pp. 345-363. Springer, Heidelberg (2007)

13. Modified Jolden and Demo (May 2008), http: / /www. cs. unm. edu/ marron

14. Rugina, R., Rinard, M.C.: Automatic parallelization of divide and conquer algorithms. In: PPOPP (1999)

15. Sagiv, S., Reps, T.W., Wilhelm, R.: Solving shape-analysis problems in languages with destructive updating. In: POPL (1996)

16. Standard Performance Evaluation Corporation. JVM98 Version 1.04 (August 1998), http: / / www. spec.org/ jvm98 